\title{
Comparative study of Propofol and Thiopental Sodium for Gastrotomy in Rabbits
}

Mukesh Kumar Menghwar ${ }^{1}$, Ahmed Nawaz Tunio ${ }^{1}$, Ghiassudin Shah ${ }^{1}$, Habibullah Janyaro ${ }^{1}$, Abdul Salam Khoso ${ }^{1}$, Rajoo Mal ${ }^{1}$, Imtiaz Ali Ujjan $^{1 *}$, Waseem Ali Vistro ${ }^{1}$, Asad Ali Jariko ${ }^{1}$, Dilpat Rai Menghwar ${ }^{2}$ and Muhammad Azhar Memon ${ }^{1}$

1Faculty of Animal Husbandry and Veterinary Science, Sindh Agriculture University Tandojam-Pakistan 2Department of Zoology, Quaid-1-azam University Islamabad-Pakistan

*Corresponding author's email: imtiazujjan61@gmail.com

\section{Citation}

Mukesh Kumar Menghwar, Ahmed Nawaz Tunio, Ghiassudin Shah, Habibullah Janyaro, Abdul Salam Khoso, Rajoo Mal, Imtiaz Ali Ujjan, Waseem Ali Vistro, Asad Ali Jariko, Dilpat Rai Menghwar and Muhammad Azhar Memon. Comparative study of Propofol and Thiopental Sodium for Gastrotomy in Rabbits. Pure and Applied Biology. Vol. 10, Issue 4, pp1338-1344. http://dx.doi.org/10.19045/bspab.2021.100138

Received: 28/12/2020 $\quad$ Revised: 19/02/2021 Accepted: 22/02/2021

Online First: 13/03/2021

\section{Abstract}

This study showed that heart and respiratory rate of all rabbits after administration of Xylazine HCL was decreased in both treatments. The body temperature was decreased throughout the period of anesthesia with both types of anesthesia and xylazine as compared to the control values. Comparison of the both treatments showed that there was no significant difference $(\mathrm{P}=0.6258)$ found in body temperature during anesthesia. However, Xylazine $\mathrm{HCl}$ produced a mild degree of sedation within $2.33 \pm 0.25$ and $2.5 \pm 0.18$ minutes with both treatments (A and $\mathrm{B}$ ) respectively. The mean time required for induction of anesthesia after administration of different anesthetic drugs was $29 \pm 1.53$ and $56 \pm 1.93$ seconds with both treatments A and B respectively. Results showed that the induction was smooth and rapid with propofol (treatment A) than thiopental sodium (treatment B). While, duration of anesthesia was $22 \pm 1.71$ and $57.5 \pm 2.81$ minutes after induction with treatment A and B respectively. Surgical anesthesia (stage 3-plane 3) occurred at 5 to 25 minutes and (stage 3-plane 2) at 5 to 40 minutes were recorded in treatments (A and B) respectively. The mean duration of analgesia was $26.67 \pm 2.11$ and $60 \pm 1.83$ minutes with treatment with A and B respectively. Degree of analgesia and degree of muscle relaxation was deep with treatment $A$ as compared to treatment B. Recovery from anesthesia was shorter $8.17 \pm 1.05$ with treatment A than18.33 \pm 1.67 minutes treatment with B. It is concluded that propofol produced safest, rapid anesthesia in all rabbits for gastrotomy than thiopental sodium in rabbits.

Keywords: Gastrotomy; Propofol; Thiopental Sodium; Rabbits

\section{Introduction}

General anesthesia provides better analgesia than local anesthesia for any surgical procedures as it provides better insensitivity to pain, complete unconsciousness and freedom from reflex responses, good muscle relaxation and loss of motor ability [1]. Therefore, in veterinary practice popular 
anesthetic induction agent propofol is widely used to produce a smooth and fast induction of anesthesia. It is characterized by a rapid recovery and effective lack of any growing effect in continuous infusion or in repeated bolus injection [2]. It inhibits the N-methyld-aspartate receptor and modulates calcium influx through slow calcium-ion channels by directly activates gamma-aminobutyric acid receptors [3]. Cardiopulmonary effects are stable when administration the continuous infusion rate of propofol for maintenance of anesthesia in donkeys [4].

Thiopental sodium is an ultrashort acting barbiturate; that possesses muscle relaxant and poor analgesia properties [5]. Whereas, it has high lipid solubility usually it is prepared just before surgery because it is unstable and strongly alkaline solution. Thiopental sodium produces unconsciousness with intravenous $(3-5 \mathrm{mg} / \mathrm{kg})$ as a $2.5 \%$ solution in $15-20$ Seconds. It is gradually metabolized and responsible to accumulate in body fats if given frequently and may have a prolonged [6]. Xylazine hydrochloride is a $\alpha_{2-}$ adrenergic agonist and it is a thiazine derivative that has sedative, muscle relaxant and analgesic effect. It is frequently used for sedation, diagnostic and minor surgical procedures in rabbits [1].

\section{Materials and Methods}

The experimental study was conducted on White New Zealand rabbits of both sexes (Male and female) with age ranges from 8 to 12 months and body weight ranging from $1000-1800 \mathrm{~g}$. Healthy rabbits were procured from the animal house of Dow International Medical hospital Ojha campus Karachi. Rabbits were housed in a separate cage, green grass and water were made available at $a d$ libitum. Total 12 animals were randomly divided into two groups placing 6 animals in each (A and B). In group A, rabbits were anesthetized with propofol 1\% (Sandoz company Austrilia) $12 \mathrm{mg} / \mathrm{kg}$ and in group B, rabbits were anesthetized with thiopental sodium (Pentothal sodium 500mg, Abbott Laboratories Karachi) $12 \mathrm{mg} / \mathrm{kg}$ after premedication with Xylazine (Xylaz 20mg/ml, Farvet company lahore) $10 \mathrm{mg} / \mathrm{kg}$ body weight. During this treatment we performed laparotomy for gastrotomy in rabbits.

Physiological parameters included: -Rectal temperature (RT), respiration rate $(\mathrm{RR})$ and heart rates (HR) were recorded on the control, at induction of pre-anesthetic agent, at induction of general anesthetic agents and every 5 minutes of interval after treatment for 60 minutes. The following parameters were measured during this experiment for all the groups: Induction time and its nature, degree, duration and onset of sedation, surgical anesthesia, recumbency, duration of recovery, standing time, degree of analgesia, degree of muscle relaxation and eye reflex. The induction and maintenance of sedation, analgesia and anesthesia was assessed according to the rabbit's responds to a painful stimulus (toe pinching). All the recorded data of both groups were tabulated and computed with mean and standard error and that further subjected to standard statistical analysis using one way analysis of variance and statistical difference between both treatments were calculated. The difference was considered significant at the level of $\mathrm{P}<0.05$ significance using SPSS. V. 21 (IBM, Inc., USA).

\section{Results}

The mean $( \pm \mathrm{SE})$ control values of heart rate in rabbits with A and B were 193.33 \pm 16.05 and $185.33 \pm 5.60$ respectively. After administration of Xylazine HCL heart rate was decreased significantly in all animals in both treatments and also decreased significantly after induction of anesthesia from the pre-anesthetic values recorded in group-A, but in group-B heart rate increased at 10 to 35 minutes after induction of anesthesia significantly from the preanesthetic values. Comparison of the two treatments showed that the heart rate after 5 
minutes of induction decreased with treatment $\mathrm{A}$ and increased up to 35 minutes and then decreased with treated $B$ after induction of anesthesia. The mean control respiratory rate $(\mathrm{RR})$ of rabbits with both treatments $\mathrm{A}$ and $\mathrm{B}$ were $72.33 \pm 1.89$ and $59.33 \pm 2.61$ breaths/minutes respectively. These values were significantly different from each other. The reduction of respiratory rate was seen after the administration of xylazine. After 5 minutes of induction of anesthesia, respiratory rate decreased significantly from control values with both treatments. The maximum decreases were recorded at 50 minutes with treatment $\mathrm{A}$, at 40 minutes with treatment $\mathrm{B}$. The mean control values of rectal temperature of rabbits with both treatments $A$ and $B$ were $101.9 \pm 0.32$ and $101.57 \pm 0.22$ respectively. The temperature was decreased after the administration of xylazine as well as both types of anesthesia such as treatment A and B compared to the control values. The body temperature was decreased throughout the period of anesthesia with both treatments in all animals. Comparison of the both treatments showed that there was no significant difference found in the body temperature during anesthesia and recovery period.

Xylazine $\mathrm{HCl}$ produced a mild degree of sedation within $2.33 \pm 0.25$ and $2.5 \pm 0.18$ minutes with both treatments $\mathrm{A}$ and $\mathrm{B}$ respectively. All the animals went on lateral recumbency within 3 minutes after Xylazine treatment. Premedication with Xylazine produced a mild degree of sedation $(++)$ with both treatments in all animals. The mean time required for induction of anesthesia after administration of different anesthetic drugs was $29 \pm 1.53$ and $56 \pm 1.93$ seconds with both treatments $\mathrm{A}$ and $\mathrm{B}$ respectively. The induction was smooth, rapid and excitement free with propofol than thiopental sodium. The mean duration of anesthesia was $22 \pm 1.71$ and 57.5 \pm 2.81 minutes after induction respectively. Surgical anesthesia (stage 3plane 3) at 5-25 minutes and (stage 3-plane 2) at 5-40 minutes were observed in treatments $\mathrm{A}$ and $\mathrm{B}$ respectively. The mean duration of analgesia was 26.67 \pm 2.11 and $60 \pm 1.83$ minutes after the administration of $\mathrm{A}$ and $\mathrm{B}$ anesthetic treatment respectively. The duration of analgesia was longer with treatment B as compared with treatment A. Degree of analgesia and degree of muscle relaxation was deep with treatment $A$ as compared to treatment B. Both anesthetic regimens produced surgical anesthesia with adequate muscle relaxation. Recovery from anesthesia was safe and excitement free with both treatments. The mean recovery period with treatment $A$ and $B$ were $8.17 \pm 1.05$ and $18.33 \pm 1.67$ minutes respectively. Duration of recovery with treatment $A$ is shorter than treatment with B. there was significantly difference between both treatment.

\section{Discussion}

This study was conducted on White New Zealand rabbits and two different anesthetic regimens were used to perform the surgery. The fundamental goal of this study was to immobilize the animal for unconscious state, analgesia and with rapid return with free of excitation [7]. In this study 12 healthy rabbits were divided into two groups (Group A and B). Group A, was treated with propofol $1 \%$ at $12 \mathrm{mg} / \mathrm{kg}$ and group B, was treated with thiopental sodium $5 \%$ at $12 \mathrm{mg} / \mathrm{kg}$ after premedicated with xylazine $\mathrm{HCl} 2 \%$ at $10 \mathrm{mg} / \mathrm{kg}$ body weight. During this study there were no any rabbits died in all experiments. Various parameters were used to assess the anesthesia and wound healing such as heart rate, respiratory rate, body temperature, induction of anesthesia, duration of anesthesia and recovery time and others observation.

Results of present study obtained after administration of propofol at $12 \mathrm{mg} / \mathrm{kg}$ (Group A), heart rate was significantly decreased in all animals. Whereas, rabbits treated with thiopental sodium at $12 \mathrm{mg} / \mathrm{kg}$ 
(Group B), heart rate was increased as compare to corresponding to control values. These findings are similar with the results of [8]. who observed that propofol $10 \mathrm{mg} / \mathrm{kg}$ had decreased heart rate. The significant increase in heart rate following induction of anesthesia with using thiopental sodium+xylazine in this study was in agreement with findings reported by [9]. Our results are agreement with the findings of [5] also reported that significant increase in heart rate after induction of anesthesia with thiopental sodium $20 \mathrm{mg} / \mathrm{kg}$ in New Zealand rabbits.

The cardiopulmonary effects of propofol as the sole anesthetic agent administered as a continuous intravenous infusion in New Zealand White rabbits [10]. Furthermore, in our results heart rate also significantly decreased when using Xylazine $\mathrm{HCl}$ these findings agreed with the results of $[11,12]$ who reported that bradycardia and decreased cardiac output cause by xylazine.

In this study respiratory rate of rabbits were significantly $(\mathrm{P}<0.05)$ decreased from baseline in both groups with the time of anesthesia induction to the time of recovery. However, similar findings were reported by [13] who observed the decrease in respiratory rate with all treatments during the anesthesia. While, [14] reported that respiratory rate was significantly reduced with propofol. The result of this study in group A, treated with propofol at $12 \mathrm{mg} / \mathrm{kg}$, there were significant decrease in respiratory rate between 5 to 50 minutes due to the depression of central nervous system by propofol lead to depression in muscular activity, then reducing the air flow rate this fact is reported by [15]. However, the group B, (thiopental sodium) exposed a significant reduced in respiratory rate at period from 10 to 40 minutes in contrast with control time. Our results are agreement with the results of [16] where the degree of respiratory depression with propofol was similar to that of thiopental sodium at 5 to 40 minutes. According to [17] who reported that thiopental sodium has a depressing effect on respiratory rate because of its direct depressing effect on the respiratory center. However, [9] showed that the respiratory rate significant decrease immediately after the injection of thiopental sodium pre-medicated with Xylazine and continued for up to 50 minutes post induction of anesthesia.

In this study body temperature in all rabbits after anesthesia, non-significant change was observed after induction of propofol and thiopental sodium. The findings of this study are agreed with [18] where they also found the decreased in rectal temperature was non significantly in goat study. [5] who reported there was no significant change in rectal temperature after injection of thiopental sodium in New Zealand Rabbits. Furthermore, [19] they did not found any difference in body temperature in rabbits with different anesthetic protocol. The physiological effects of the anesthetic drugs caused a reduction in rectal temperature during surgery. Also, poor physical health of the patient, incision large surface area deep anesthesia, long surgical trials, infusion of cold fluids intravenously and a low environmental temperature [15] have been implicated. In this study, a wide area of the skin from (xypoid to the umbilicus) was cut may have contributed to the hypothermia. In results of ongoing study on rabbits showed that rabbits pre-medicated with Xylazine at $10 \mathrm{mg} / \mathrm{kg}$ produced sedative, anesthetic effect and a mild degree of sedation. Our results are agreement with the study of [20] they observed during induction of anesthesia between 5 to 40 minutes with significant deep analgesia and muscle relaxation. Reduced to moderate analgesic effect and muscle relaxation at 15 minutes in propofol, thiopental sodium at 5 to 20 minutes, then low to mild analgesia and muscle relaxation at 35 minutes in propofol and thiopental 
sodium and xylazine between 20 to 60 minutes these results are in agreement with [5] The combinations of xylazine $10 \mathrm{mg} / \mathrm{kg}$ with Propofol and thiopental sodium cause increase the effect of analgesia.

In present study initial time of induction of anesthesia was $29 \pm 1.53$ second in all rabbits observed after administration of propofol, while late time of induction of anesthesia $56 \pm 1.93$ second recorded in all rabbits with thiopental sodium. These results are similar that of propofol cause quick induction time appears by suggesting [21] and rapid cross of the drug to the blood brain barrier because of its lipid solubility. This comparison revealed that the induction of anesthesia using thiopental sodium with xylazine caused significantly longer duration of anesthesia [9]. It is reported a significant decrease in initiation time because of increased in thiopental sodium dose. These reports are supporting our finding which showed that the increase of thiopental sodium dose resulted in a parallel increase in anesthetic phase [22].

In the current study longer duration of anesthesia 57.5 \pm 2.81 minutes was recorded after administration of thiopental sodium (Group B), while the shortest duration of anesthesia was $22 \pm 1.71$ minutes noted in group A, after administration of propofol in all rabbits. The findings of longer duration are agreement with the results of [22] that were found usually thiopental sodium to have longer periods of anesthetic induction. Similarly, these results are in agreement with the findings of [18] who reported a significant with longer anesthetic induction, when anesthesia was induced with thiopental sodium in goat kids.

In this study after administration of propofol, surgical anesthesia (Stage 3-Plane 3) was noted in rabbits group A, while (stage 3 plane 2) was observed after administration of thiopental sodium in rabbits group B. This consistent with the finding [13] study propofol anesthesia for a maintaining was considered as a better then another. Propofol caused hydroxylation along with glucuronide and sulphate in the liver [23] Propofol is quickly redistributed due to lipophilic property [15], which shows the fast development in propofol induction.

The regaining time on the pedal reflex, palpebral reflex, tongue reflex, jaw reflex, cough reflex and swallowing reflex were monitored during these experiments. All reflexes did not show any significant differences in their regaining time throughout the course of anesthesia, which was induced using both anesthetic agents. These results are also supported the findings of [24] who reported that, the eyes usually remain open and pupils were dilated in animals anesthetized with thiopental sodium. [24] who reported that palpepral reflex persisted did not affect through all anesthetic protocols and longer mean time of depression of tongue reflex was seems with thiopental sodium.

Significantly longer recovery time from anesthesia was noted where using thiopental sodium $18.33 \pm 1.67$ minutes, while shorter with propofol $8.17 \pm 1.05$ minutes of anesthesia recorded in all rabbits. Whereas, all these results are agreed with the results of [25] who reported that increased recovery time with thiopental sodium administration. Propofol caused in rapid induction within 2023 seconds and smooth recovery with total recumbency periods of anesthesia [14]. Although propofol has a fast onset and smooth, quick recovery might be due to fast redistribution and rapid metabolism found by [16] Total recovery time is the most important factors leading the selection of anesthetic protocols for the different surgical procedure and many surgeons prefer the use of anesthetic drug that can confirm at is factory duration of anesthesia for a surgical procedure with a fast, smooth and ordinary recovery from anesthesia.

\section{Conclusion}


It is concluded that on the basis of present results Propofol produced safest, rapid anesthesia in all rabbits for gastrotomy than thiopental sodium in rabbits. Propofol scored superior than thiopental sodium because of smooth induction and safer recovery from anesthesia in rabbit model. Thiopental sodium had some disadvantages like tachycardia and longer duration of recovery.

\section{Authors' contributions}

Conceived and designed the experiments: M Kumar, AN Tunio, G Shah, H Janyaro, AS Khosa, R Mal \& IA Ujjan, Performed the experiments: M Kumar \& AN Tunio, Analyzed the data: M Kumar, AN Tunio \& WA Vistro, Contributed reagents/ materials/ analysis tools: M Kumar, AN Tunio, \& MA Memon, Wrote the paper: M Kumar.

\section{References}

1. Thurman JC, Tranquily WJ \& Benson GJ (1999). Essentials of Small Animal Anesthesia. Lippincot, William and Wilkins, Philadelphia, USA. pp. 382404.

2. Adetunji A, Ajadi RA, Adewoye CO \& Oyemakinde BO (2002). Total intravenous anesthesia with Propofol: Repeated bolus versus continuous Propofol infusion technique in Xylazinepremedicated dogs. Int J Vet Med 57: 139-149.

3. Kotani Y, Shimazawa M, Yoshimura S, Iwama $\mathrm{T} \&$ Hara H (2008). The Experimental and Clinical Pharmacology of Propofol, an Anesthetic Agent with Neuroprotective Properties. CNS Neurosci and Therap 14(2): 95-106.

4. Naddaf H, Baniadam A, Rasekh A, Arasteh A \& Sabiza B (2015). Cardiopulmonary effects during anesthesia induced and maintained with propofol in acepromazine pre-medicated donkeys. Vet Anesthesia Analgesia 42(1): 83-87.
5. Mohammed AA, Sayed MAM \& Abdelnabi MA (2011). A New Protocol of Anesthesia Using Thiopental, Diazepam and Xylazine in White New Zealand Rabbits. Australian J of Basic and Appl Sci 5(9): 1296-1300.

6. Gin T, Mainland P, Chan MT \& Short TG (1997). Korean Journal of Animal Anaesthesiology. 86(1): 73-78.

7. Garcia AA, Sumano H \& Nunez E (2002). Pharmacologic basis of shortterm intravenous general anesthesia in equine. Vet México 33(3): 309-333.

8. AsaAkine, Suzuka H, Hayashida Y \& Kato Y (2001). Effects of ketamine and propofol on autonomic cardiovascular function in chronically instrumented rats. J Elsev Sci Basic Vet 87(2): 201208.

9. Nuha MO (2004). Evaluation of selected anesthetic protocols for total intravenous anesthesia in goat kids undergoing laparotomy. M.Sc. Thesis, faculty of Veterinary Science, U of K. Ind J Anim Sci 85(11): 1279-1284).

10. Aeschbacher G \& Webb AI (1993). Propofol in rabbits. 2. Long term anesthesia. J Lab Anim Sci 43(4): 32835.

11. Kul M, Koc Y, Alkand F \& Ogurtan Z (2000). The effects of XylazineKetamine and Diazepam-Ketamine on arterial blood pressure and blood gases in dogs. On J Vet Res 4: 123-132.

12. Ismail $\mathrm{Z}$, Jawasreh $\mathrm{K}$ \& Al-Majali A (2010). Effects of xylazine-ketaminediazepam anesthesia on certain clinical and arterial blood gas parameters in sheep and goats. J Comparat Clinic Pathol 19(1): 11-14.

13. Carregaro (2016). Propofol application in veterinary sedation and anesthesia $J$ Lab Anim Prac 17(5): 4.

14. Abu-Ahmed MH (2015). Assessment of clinical anesthesia and cardiopulmonary effects of Propofol in Metomidine 
premedicated donkeys. Journal Article: Res Opin Ani And Veter Sci 5(1): 38-42.

15. Adetunji A, Ajadi RA, Adewoye $\mathrm{CO} \&$ Oyemakinde BO (2002). Total intravenous anesthesia with Propofol: Repeated bolus versus continuous Propofol infusion technique in Xylazinepremedicated dogs. Int J Vet Med 57: 139-149.

16. Flaherty D (2009). Anesthetic DrugsChapter 6. Anesthesia for Veterinary Nurses. 1st edition, Edited by: Welsh, L.; Wiley - Blackwell, A John Wiley \& Sons, Ltd., pp 121-161.

17. Raffal AO, Aziz OK, Eesa MJ \& Abed BS (2017). Comparative study for three protocols of general anesthesia in bucks. Iraq J Vet Med 41(2): 15-23

18. Ghurashi MAH (1999). Some aspects of short term Thiopentone sodium anesthesia with or without selected preanesthetic medication in goat kid. Thesis, faculty of Veterinary Science, University of Khartoum.

19. Jasim D, Hankle J \& Brill (2011). Comparative study of different intramuscular anesthesia combination in rabbit. J Veter Anesth and Analg, 34: 274-280.
20. Ahmed A, Al-Qarwa AA \& Musa AM (2014). Aphradynamic study of propofol and ketamine infusion in ponies undergoing surgery. J Res Vet Sci 64: 159-164.

21. Grint NJ \& Murison PJ (2008). A comparison of Ketamine-Midazolam and Ketamine-Medetomide combinations for induction if anesthesia in rabbits. $J$ Veter Anesth and Analg 35 (2): 113-121.

22. Radi MAA, Seri HI \& Ghurashi MAH (2011). Effect of Thiopentone sodium on some physiological and anesthetic parameters in donkeys. Suez Canal Veter Med J 14(1): 81-88.

23. Horn E \& Nesbit SA (2004). Pharmacology and pharmacokinetics of sedatives and analgesics. Gastrointestinal Endoscopy Clinics of North America 14(2): 247-182.

24. Thurmon JC, Tranquilli WJ, Benson GJ \& Lumb WV (1996). Lumb \& Jones' Veterinary Anesthesia and analgesia. 4th edition. Lippincott Williams \& Wilkins, 928.

25. Elzubair AA \& Seri HI (2015). Quality of anesthesia induced using DiazepamThiopentone Sodium in Donkeys. Sud $J$ Sci \& Technol 16(2): 15-23. 